\title{
The High Energy Behavior of Gamma-ray Burst Spectra as Observed by Fermi GBM and LAT
}

\section{Péter Veres* ${ }^{\dagger}$}

Dept. of Astronomy \& Astrophysics, Dept. of Physics and Center for Particle Astrophysics Pennsylvania State University

E-mail: veresp@psu.edu

\section{Péter Mészáros}

Dept. of Astronomy \& Astrophysics, Dept. of Physics and Center for Particle Astrophysics Pennsylvania State University

E-mail: nnpeastro.psu.edu

\begin{abstract}
Most Fermi GRB spectra appear as either a broken power law extending to $\mathrm{GeV}$ energies or as a broken power with a separate $\mathrm{GeV}$ power law component. Here we show that such spectra can be understood in terms of magnetically dominated relativistic jets where a dissipative photosphere produces the prompt $\mathrm{MeV}$ emission, which is extended into the $\mathrm{GeV}$ range by inverse Compton scattering in the external shock, with possible contributions from a reverse shock as well. The bulk Lorentz factors required in these models are in the range of 300-600, and the MeV-GeV time delays arise naturally. In some cases an optical flash and a sub-dominant thermal component are also present.
\end{abstract}

Gamma-Ray Bursts 2012 Conference -GRB2012,

May 07-11, 2012

Munich, Germany

\footnotetext{
* Speaker.

${ }^{\dagger}$ We acknowledge NASA NNX09AL40G, NSF PHY-0757155 and OTKA grant K077795 for partial support, and thank Bin-Bin Zhang, Shan Gao and Kenji Toma for useful comments.
} 


\section{Introduction}

The GRB spectra observed with the $L A T$ detector on Fermi reveal a diversity of appearances. Some have spectra consistent with a single canonical Band function [1] extending to the highest detected multi-GeV energies, while in others at the higher end of the Band spectrum a second, flatter spectral component emerges, which sometimes appears to have a cutoff [2]. Here we investigate possible scenarios for these two spectral types in the context of magnetically dominated, baryon loaded outflows, where the prompt $\mathrm{MeV}$ emission arises in the jet photosphere ([3-6]). Photospheric emission, either in baryonic or magnetic models, where dissipation occurs near the photosphere, have been shown to be able to produce Band-like non-thermal spectra [7-9]. In the scenarios that we discuss here we assume that the prompt spectrum is a combination of the emission from a magnetically dominated dissipative photosphere and the external shock. We argue that the photosphere is initially responsible for the low energy (GBM) part of the Band-like prompt spectrum, the final observed spectrum being modified by inverse Compton scattering in the external shock, which results in a delayed high energy (LAT) component. The latter includes a forward and possibly also a reverse shock contribution, dominated by self-inverse Compton (SSC) as well as external inverse Compton (EIC) of the photospheric photons. We consider here mainly the radial structure of the outflow, and restrict ourselves to leptonic mechanisms.

\section{Magnetic Model}

We assume a total luminosity $L_{t}=10^{53} L_{t, 53} \mathrm{erg} / \mathrm{s}$ is released at a radius $r_{0}=10^{7} r_{0,7} \mathrm{~cm}$, lasting for $10 \mathrm{~s}$ in the central engine frame at redshift $z=1$. This is initially (at $r_{0}$ ) mainly magnetic, which at larger radii gets gradually converted into bulk kinetic energy and non-thermal radiation. We assume that at the dissipative photosphere a fraction $\zeta_{r} \simeq 0.5$ of $L_{t}$ is released as prompt radiation. The remaining energy fraction beyond the photosphere $\zeta_{k}$, is in kinetic energy form. Our results are evaluated for $\zeta_{r}=1-\zeta_{k}=0.5$. In magnetically dominated models the Lorentz factor will increase more slowly than in a matter dominated case $\Gamma(r) \propto r^{1 / 3}$ if $r<r_{\text {sat }}$ and constant for $r_{\text {sat }}<r<r_{\text {dec }}$ where $r_{\text {sat }}$ is the saturation radius where $\Gamma \rightarrow \eta \equiv L_{t} / \dot{M} c^{2}$ and $r_{\text {dec }}>r_{\text {sat }}$ is the deceleration radius. We will concern ourselves here only with the behavior before and in the neighborhood of the deceleration radius, and do not consider the longer term afterglow phase.

\section{Prompt and Afterglow Radiation Components}

The photospheric radius is at $r_{p h}=6.5 \times 10^{12} L_{t, 53}^{3 / 5} \zeta_{r}^{3 / 5} r_{0,7}^{2 / 5} \eta_{600}^{-3 / 5} \mathrm{~cm}$. This occurs in the accelerating phase for any realistic set of parameters. The saturation radius, for a magnetically dominated jet is at $r_{s a t}=2.2 \times 10^{15} r_{0,7} \eta_{600}^{3} \mathrm{~cm}$. At the $r_{d e c}=4.8 \times 10^{16} L_{t, 53}^{1 / 3}\left(1-\zeta_{r}\right)^{1 / 3} t_{1.3}^{1 / 3} n_{0}^{-1 / 3} \eta_{600}^{-2 / 3} \mathrm{~cm}$ deceleration radius, the comoving baryon density is $n_{b, d e c}^{\prime}=5.3 \times 10^{4}\left(1-\zeta_{r}\right)^{-2 / 3} L_{t, 53}^{1 / 3} \eta_{600}^{-2 / 3} n_{0}^{2 / 3} t_{1.3}^{-2 / 3} \mathrm{~cm}^{-3}$.

\subsection{Nonthermal Photospheric Spectrum}

The value of the Lorentz factor at the magnetic photosphere is $\Gamma_{p h}=87 L_{t, 53}^{1 / 5} \zeta_{r}^{1 / 5} r_{0,7}^{-1 / 5} \eta_{600}^{-1 / 5}$. At the photosphere, the magnetic dissipation and/or collisional effects such as $n, p$ decoupling will 
lead to a significant non-thermal electron component. E.g. for reconnection acceleration or semirelativistic shocks associated with reconnection [5], a typical minimum comoving electron random Lorentz factor $\gamma_{e, p h} \sim 600 \Gamma_{r}$. The magnetic field at the photosphere is $B_{p h}^{\prime}=1.7 \times 10^{6} L_{t, 53}^{-1 / 5}(1-$ $\left.\zeta_{r}\right)^{1 / 2} \zeta_{r}^{-7 / 10} r_{0,7}^{-3 / 10} \eta_{600}^{1 / 5} \varepsilon_{B, 0}^{1 / 2} \Gamma_{r}$ G. Here $n_{b}^{\prime}$ is the comoving baryon density evaluated at the photosphere, $n_{b, p h}^{\prime}=2.0 \times 10^{13} L_{t, 53}^{-2 / 5} \zeta_{r}^{-7 / 5}\left(1-\zeta_{r}\right) r_{0,7}^{-3 / 5} \eta_{600}^{2 / 5} \mathrm{~cm}^{-3}$. The observed synchrotron spectral peak of the photosphere will be $\varepsilon_{s y}^{p h, o b s}=\varepsilon_{b r}=310 \zeta_{r}^{-1 / 2}\left(1-\zeta_{r}\right)^{1 / 2} r_{0,7}^{1 / 2} \varepsilon_{B, 0}^{1 / 2} \Gamma_{r}^{3}\left(\frac{1+z}{2}\right)^{-1} \mathrm{keV}$, corresponding to the Band peak. The magnetic field energy fraction at the photosphere is still $\varepsilon_{B} \sim 1$, but dissipation will lead to values $\varepsilon_{B, F S} \lesssim 0.1$ by the time the flow reaches the deceleration radius. Following [?] and [8], we assume that the emergent spectral shape is of the Band form. We assume $\alpha \simeq 1$ (where $N_{\varepsilon}(\varepsilon) \propto \varepsilon^{-\alpha}$ ) and for the high energy spectral index $\beta \simeq 2.4$.

We discuss two scenarios for the high energy behavior of the photospheric emission. In the first scenario, photons above the peak are upscattered as a result of interactions with electrons associated with magnetic turbulent waves, up to an energy $\varepsilon^{\prime} \approx m_{e} c^{2}$ in the jet frame [10] corresponding to a cutoff at $\varepsilon_{h} \approx 30 L_{t, 53}^{1 / 5} \zeta_{r}^{1 / 5} r_{0,7}^{-1 / 5} \eta_{600}^{-1 / 5}\left(\frac{1+z}{2}\right)^{-1} \mathrm{MeV}$. In this case no pairs are created. In the second scenario electrons can be accelerated, to a power law extending above $\varepsilon_{h}$ to sufficiently high energies for $\gamma \gamma$ interactions. These pairs also radiate in the magnetic field of the prompt emission site, peaking at tens of electron volts.

\subsection{Thermal Component of the Photospheric Spectrum}

In the presence of collisional or magnetic dissipation, in addition to a nonthermal component one expects also a thermal component with a quasi-blackbody spectrum. In our case, this peaks in the soft X-rays [5], due to the different temperature scaling with radius for the magnetic dynamics, comprising a fraction $\zeta_{t h}$ of the luminosity of the photosphere. This thermal component can be calculated from the initial $T_{0}=2.1 L_{t, 53}^{1 / 4} r_{0,7}^{-1 / 2} \Gamma_{r}^{-1 / 2} \mathrm{MeV}$ at the initial radius $r_{0}=10^{7} r_{0,7} \mathrm{~cm}$. Above $r_{0}$ the magnetically dominated jet dynamics $\Gamma \propto r^{1 / 3}$ implies a comoving volume is $V^{\prime} \propto r^{2} \Gamma \propto r^{7 / 3}$ in the acceleration regime. Thus, the temperature will decrease with radius as $T^{\prime} \propto \rho^{\prime \hat{\gamma}-1} \propto \rho^{\prime 1 / 3} \propto V^{\prime-1 / 3} \propto r^{-7 / 9}$, or $T^{\prime}(r)=T_{0}\left(r / r_{0}\right)^{-7 / 9}$, where $\hat{\gamma}=4 / 3$ is the adiabatic exponent. Thus, at the photosphere $T\left(r_{p h}\right)=2.7 L_{t, 53}^{-1 / 60} \zeta_{r}^{-4 / 15} \eta_{600}^{4 / 15} r_{0,7}^{-7 / 30} \Gamma_{r}^{-1 / 2}\left(\frac{1+z}{2}\right)^{-1} \mathrm{keV}$. The corresponding thermal luminosity is $L_{t h}=6.5 \times 10^{49} \mathrm{erg} / \mathrm{s}$, which is low for producing the prompt emission, as well as being too soft. This blackbody component from the photosphere thus peaks in the soft $\mathrm{X}$-rays at $\left(\varepsilon F_{\varepsilon, B B}\right)^{\text {peak }} \approx 9.5 \times 10^{-9} L_{t, 53}^{11 / 15} \zeta_{r}^{-4 / 15} \eta_{600}^{4 / 15} r_{0,7}^{4 / 15} \mathrm{erg} \mathrm{cm}^{-2} \mathrm{~s}^{-1}$. This thermal component is similar to the one found by [11].

\subsection{Forward- and Reverse Shock (FS, RS) Synchrotron}

The forward shock is expected to develop for all parameters. The presence of the reverse shock depends on the magnetization parameter at $r_{\mathrm{dec}}$. Without going into details, we mention, that the FS will be in the fast cooling regime for most of the parameter space and the reverse shock will be in the slow cooling phase. We follow the treatment of [12]. The peak flux density of the FS synchrotron spectrum occurs, at $F_{\max }^{F S}\left(\varepsilon_{c}\right)=0.15 L_{t, 53}\left(1-\zeta_{r}\right) t_{1.3} \varepsilon_{B,-1}^{1 / 2} n_{0}^{1 / 2} D_{L, 28.3}^{-2}$ Jy. The scattering optical depth in the FS is given by $\tau_{F S}=1.1 \times 10^{-8} L_{t, 53}^{1 / 3}\left(1-\zeta_{r}\right)^{1 / 3} t_{1.3}^{1 / 3} \eta_{600}^{-2 / 3} n_{0}^{2 / 3}$. The peak flux density of the RS is $F_{\text {max }}^{R S}\left(\varepsilon_{m}\right)=92 L_{t, 53}\left(1-\zeta_{r}\right) t_{1.3} \eta_{600} \varepsilon_{B,-1}^{1 / 2} n_{0}^{1 / 2} D_{L, 28.3}^{-2} \mathrm{Jy}$. The optical depth of the 
RS at $r_{d e c}$ is $\tau_{R S}=6.4 \times 10^{-6} L_{t, 53}^{1 / 3}\left(1-\zeta_{r}\right)^{1 / 3} t_{1.3}^{1 / 3} \eta_{600}^{1 / 3} n_{0}^{2 / 3}$. The synchrotron self Compton emission of the FS and of the RS (FS-SSC, RS-SSC) is also expected to be present.

\subsection{EIC scattering of photospheric nonthermal photons on external forward and reverse shock electrons (FS-EIC, RS-EIC)}

The electrons in the forward shock will also lose energy by external inverse Compton (EIC) as they upscatter the photons from the prompt emission (photospheric) region. A rough estimate of the peak of the EIC emission is $\left(\varepsilon F_{\varepsilon}\right)_{E I C}^{\text {peak }} \sim 7.2 \times 10^{-10} L_{t, 53} \zeta_{r}^{3 / 2}\left(1-\zeta_{r}\right)^{-1 / 2} \varepsilon_{B, 0}^{-1 / 2} \varepsilon_{B,-1}^{-1} \Gamma_{r}^{-3}(1+$ $Y)^{-1} D_{L, 28.2}^{-2} \mathrm{erg} \mathrm{cm}^{-2} \mathrm{~s}^{-1}$. If the reverse shock develops, there will be an external inverse Compton component from the reverse shock electrons scattering prompt photons as well. The peak of the emission will be at the Klein Nishina cutoff frequency for this component. The peak flux of the emission is $\varepsilon F_{\varepsilon, R S E I C}^{\mathrm{peak}} \approx 2.3 \times 10^{-8} L_{t, 53}^{4 / 3} \zeta_{r}^{3 / 2}\left(1-\zeta_{r}\right)^{-1 / 6} t_{1.3}^{1 / 3} \eta_{600}^{4 / 3} n_{0}^{2 / 3} \Gamma_{r}^{3} r_{0}^{-1 / 3} \varepsilon_{B, 0}^{-1 / 2} \varepsilon_{e,-2} g_{p, 2.4} D_{L, 28.2}^{-2} \mathrm{erg} \mathrm{cm}^{-2} \Omega^{1}$.

\section{Discussion}
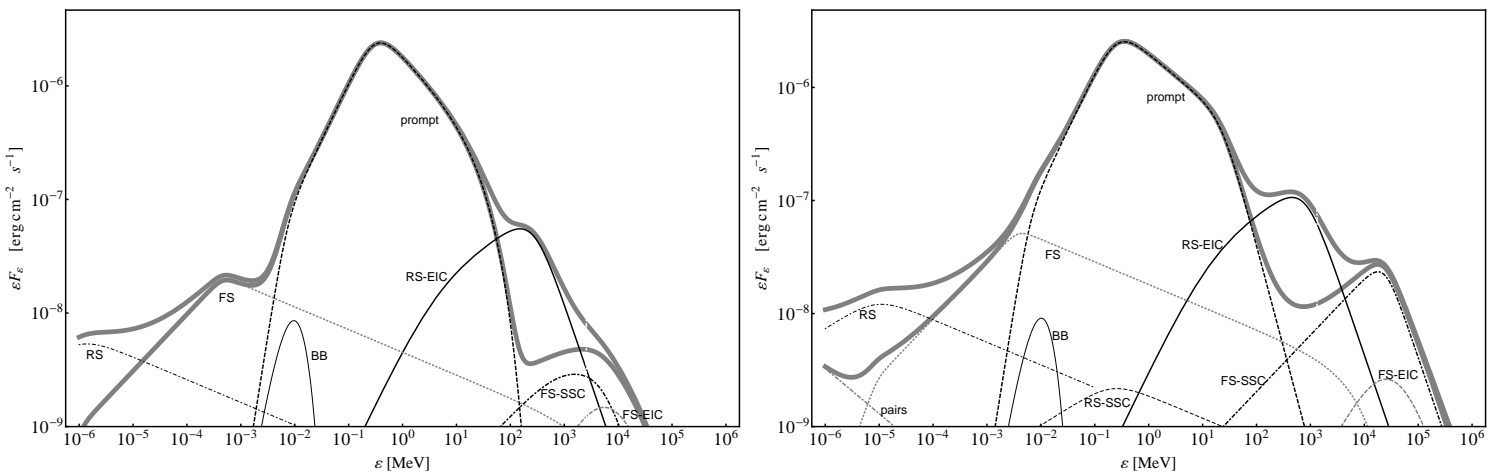

Figure 1: A model without/with pair formation (left/right).The parameters are $L_{t}=10^{53} \mathrm{erg} / \mathrm{s}, t=20 \mathrm{~s}, \zeta_{r}=$ $0.5 / 0.6, \zeta_{k}=0.5 / 0.4, n=30 / 10 \mathrm{~cm}^{-3}, \eta=400 / 600, \varepsilon_{B, p r}=1, \varepsilon_{B, F S}=\varepsilon_{B, R S}=2 \times 10^{-2} / 10^{-2}, \varepsilon_{e, F S}=$ $\varepsilon_{e, R S}=5 \times 10^{-3} / 10^{-2}, r_{0}=10^{7} \mathrm{~cm}, z=1, \beta=2.5, p=2.4$. The black dashed line is the prompt synchrotron emission, black thin continuous line is the prompt thermal component (marked BB), the thick black line is the RS-EIC, the gray, thick, dotted line is the forward shock synchrotron part (FS), the gray, dashed line is the forward shock external inverse Compton, the black dash-dotted is the FS-SSC component and the gray, dash-dotted is the RS synchrotron component and the thick dash-dotted is the pair synchrotron contribution. The thick gray continuous line is the sum of the components (the upper one is with- and the lower one without the RS contributions).

Here we show two examples (Figure 1) representative of the cases with and without pair formation. We find that, qualitatively, these models are able to explain the observed diversity of GBM and $L A T$ prompt emission spectra, without invoking an internal shock emission at intermediate radii. The presence or relative strength of a reverse shock plays a role in determining the spectral shape and the basic dichotomy in the latter. As discussed by, e.g. [13-15], for modest magnetization $\sigma$ of the outflow a reverse shock may be expected; and while for initially magnetically dominated outflows $\sigma$ (or $\varepsilon_{B}$ ) is still large at the photosphere, at larger radii it can be expected to drop sufficiently low to make reverse shocks possible, although uncertainties remain concerning 
the threshold magnetization and the reverse shock strength. When a reverse shock is present, its inverse Compton radiation can result in a larger fluence extra high energy component, 0.5-1 orders of magnitude below the Band fluence; or it can result in a smooth continuation of the Band component, which can mimic a single Band high energy component of relatively hard effective slope $\beta$. The fact that the LAT emission is contributed by the external shock implies that it will appear with an intrinsic delay of order $t_{d e l} \sim r_{d e c}(1+z) / c \eta^{2} \approx 4.4 L_{t, 53}^{1 / 3}\left(1-\zeta_{r}\right)^{1 / 3} t_{1.3}^{1 / 3} n_{0}^{-1 / 3} \eta_{600}^{-8 / 3}(1+z) / 2 \mathrm{~s}$, of order a few seconds relative to the photospheric $\mathrm{MeV}$ component. Such a delay was indicated also in some early work on LAT spectra $([16,17])$ interpreted as FS synchrotron emission. Here, the dominating components come from the inverse Compton mechanism. The implications of our model for constraints on the bulk Lorentz factor are much less stringent than in one-zone models where the $G B M$ and $L A T$ emission are assumed to arise in the same region. In our two-zone model the high energy photons arise in the external shock, the spectra can be reproduced with terminal Lorentz factors $\Gamma \sim \eta \sim 300-600$.

In our magnetized models, in the absence of pair formation this component cuts off above $\sim 50 \mathrm{MeV}$, or in the presence of pair formation, it steepens by one power law index above $\sim$ $100 \mathrm{MeV}$. Recently [18] analysed the paucity of GRBs measured by LAT and nearly half of the bursts required a break under $0.1 \mathrm{GeV}$ to explain the nondetection by LAT. In the framework of this model this is either due to a softening because of pair creation or a cutoff according to the magnetic acceleration mechanism.

The magnetic photosphere models also predict a weaker thermal component peaking at a few keV, plotted in Fig. 1. Such a thermal component has been reported in [19] for GRB 100724B. In our models such a component appears at approximately the right energies, its fluence generally being low compared to the nonthermal components. However, only for a relatively small range of parameters would it appear possible to detect it. One problem is that it can be conflated with the forward shock synchrotron component.

Another interesting component is the optical band extension of the external shock synchrotron spectra (see Fig. 1). It is seen that just the external forward shock by itself already can produce optical flashes of $m_{R} \gtrsim 12$ (Fig. 1). If pair formation occurs in the photosphere, the cooled pairs there can lead to flashes of $m_{R} \sim 9.3$ (Fig. 1) or even brighter for suitable sets of parameters. Generically, the relative scarcity of observed optical flashes may be attributed to the fact that their brightness scales roughly the same way as the prompt GeV luminosity $\left(\left(\varepsilon F_{\varepsilon}\right)_{\text {peak }}^{R S} \propto L_{t}^{p / 3} \propto L_{t}^{0.8},\left(\varepsilon F_{\varepsilon}\right)_{\text {peak }}^{\text {prompt }} \propto\right.$ $L_{t}$ and $\left.\left(\varepsilon F_{\varepsilon}\right)_{\text {peak }}^{R S-E I C} \propto L_{t}^{4 / 3}\right)$, as well as to the fact that reverse shocks may be rare in magnetically dominated outflows.

For the naked eye GRB 080319, the roughly similar behavior of the optical and $\gamma$-ray light curves can be used to argue for a common origin [20]. A fast variability of the prompt optical flash might be suggestive of an origin in the same region as the prompt $\mathrm{MeV}$ emission, which might be attributed to the pair synchrotron component. However, only very rare parameter combinations could push the optical flux of the pair synchrotron component up to $m_{R} \sim 5$ as in the naked eye burst [20]. E.g. in our model a combination of $\eta \sim 1000, \beta \approx 2.1$ and $\zeta_{r} \approx 0.99$ would approach such brightnesses.

We note that this model may be applicable both to long and short bursts, since a magnetized photosphere and an external shock would be expected in both cases. The relatively shorter $\mathrm{GeV}-\mathrm{MeV}$ delays in the short burst cases could be understood in terms of a closer-in deceleration 
or a larger Lorentz factor, the latter being suggested also by their harder $\mathrm{MeV}$ spectra.

\section{References}

[1] Band, D. et al. BATSE observations of gamma-ray burst spectra. I - Spectral diversity ApJ, 413 (1993) 281

[2] Pe'er, A. The Impact of Fermi on the Study of Gamma-ray Bursts (2011) [astro-ph/1111.3378]

[3] Drenkhahn, G., \& Spruit, H. C. Efficient acceleration and radiation in Poynting flux powered GRB outflows A\&A, 391 (2002) 1141, [astro-ph/0202387]

[4] McKinney, J. C., \& Uzdensky, D. A. A reconnection switch to trigger gamma-ray burst jet dissipation MNRAS, 1766 (2011) [astro-ph/1011.1904]

[5] Mészáros P., \& Rees, M. J. GeV Emission from Collisional Magnetized Gamma-Ray Bursts ApJL, 733 (2011) L40, [astro-ph/1104.5025]

[6] Bošnjak, Ž., \& Kumar, P. Magnetic jet model for GRBs and the delayed arrival of $>100 \mathrm{MeV}$ photons MNRAS, L390 (2012) [astro-ph/1108 . 0929]

[7] Pe'er, A., Mészáros , P., \& Rees, M. J. The Observable Effects of a Photospheric Component on GRB and XRF Prompt Emission Spectrum ApJ, 642 (2006) 995, [astro-ph/0510114]

[8] Beloborodov, A. M. Collisional mechanism for gamma-ray burst emission MNRAS, 407 (2010) 1033, [astro-ph/0907.0732]

[9] Ryde, F. et al. Identification and Properties of the Photospheric Emission in GRB090902B ApJL, 709 (2010) L172, [astro-ph/0911.2025]

[10] Thompson, C. A Model of Gamma-Ray Bursts MNRAS, 270 (1994) 480

[11] Page, K. L. et al. GRB 090618: detection of thermal X-ray emission from a bright gamma-ray burst 2011, MNRAS, 416 (2011) 2078

[12] Sari, R., Piran, T., \& Narayan, R. Spectra and Light Curves of Gamma-Ray Burst Afterglows 1998, ApJL, 497 (1998) L17, [astro-ph/9712005]

[13] Narayan, R., Kumar, P., \& Tchekhovskoy, A. Constraints on cold magnetized shocks in gamma-ray bursts 2011, MNRAS, 416 (2011) 2193, [astro-ph/1105.0003]

[14] Mimica, P., \& Aloy, M. A. Efficiency of internal shocks in magnetized relativistic jets (2011) [astro-ph/1111.3170]

[15] Giannios, D., Mimica, P., \& Aloy, M. A. On the existence of a reverse shock in magnetized gamma-ray burst ejecta A\&A, 478 (2008) 747, [ast ro-ph/ 0711.1980 ]

[16] Ghisellini, G., Ghirlanda, G., Nava, L., \& Celotti, A. GeV emission from Gamma Ray Bursts: a radiative fireball? (2009) [ast ro-ph/0910.2459]

[17] Kumar, P., \& Barniol Duran, R. On the generation of high-energy photons detected by the Fermi Satellite from gamma-ray bursts MNRAS, 400 (2009) L75, [astro-ph / 0905 . 2417]

[18] Kocevski, D., \& The Fermi Collaboration. Constraining the High-Energy Emission from Gamma-ray Bursts with Fermi (2012) [astro-ph/1201.3948]

[19] Guiriec, S. et al. Detection of a Thermal Spectral Component in the Prompt Emission of GRB 100724B ApJL, 727 (2011) L33, [astro-ph/1010 . 4601]

[20] Racusin, J. L. et al. Broadband observations of the naked-eye $\gamma$-ray burst GRB080319B Nature, 455 (2008) 183, [astro-ph/0805.1557] 